\title{
Fungal Lipids: The Biochemistry of Lipid Accumulation
}

\author{
A. Akpinar-Bayizit
}

\begin{abstract}
Current interest in single cell oils (SCOs) accumulated by oleaginous fungi centers around the ability of these microorganisms to convert agro-industrial surpluses and residues into lipids as potential alternative to edible plant and/or animal lipids or rarely found in the plant or animal kingdom [i.e. lipids containing polyunsaturated fatty acids (PUFAs)]. In a recent development, SCOs are considered as adequate starting material for the production of biodiesels. PUFAs are valuable products because of their health promoting roles. Production of microbial PUFAs have many advantages, such as short life cycle, less labor requires, less affection by venue, season aclimateasier to scale up. The main aim of this review was to explain the biochemistry behind lipid accumulation in fungi and current applications of fungal PUFAs.
\end{abstract}

Index Terms - Single cell oil, polyunsaturated fatty acids, fermentation, fungi.

\section{INTRODUCTION}

Oils and fats are both classed as lipids, substances of vegetable and animal origin that widely found in nature and form the third major group of macronutrients after proteins and carbohydrates [1]. Although lipid analysts tend to have a firm understanding of what is meant by the term "lipid", there is no widely-accepted definition. General text books usually describe lipids as a group of naturally occurring compounds, which have in common a ready solubility in such organic solvents as hydrocarbons, chloroform, benzene, ethers and alcohols [2]-[4]. A more specific definition of lipids than one based simply on solubility is necessary, and most scientists do restrict the use of "lipid" to fatty acids and their naturally-occurring derivatives (esters or amides) [5], [6]. Lipids have many metabolic roles, for example, they act as storage materials in animals, plant and microbial cells and are also responsible for the structure of cell membranes, and protect the body against cold and other environmental influences, etc. One of their important physiological roles is that they are precursors of hormone-like compounds, which is performedmainly by polyunsaturated fatty acids [7], [8].Though the human body can produce saturated and mono-unsaturated fatty acids from food components, it cannot synthesize polyunsaturated fatty acids. In other words, these have to be supplied externally from specific foodstuffs such as leafy vegetables and fish. This explains why polyunsaturated fatty acids are called "essential fatty acids" [9].

Approximately $80 \%$ of the world's oil and fat need is

Manuscript received November 20, 2013; revised February 25, 2014.

A. Akpinar-Bayizit is with the Department of Food Engineering, Faculty of Agriculture, Uludag University, Bursa, Turkey (e-mail: abayizit@uludag.edu.tr). derived from agricultural products, and the remainder coming from animal and marine sources. It is essential to find new sources for oil and protein supplement with concern to the nutritional problems accompanying the rapid growth of world's population. In fear of the depletion of oil resources and the global warming, present biotechnological research has concentrated on the commercial exploitation of microorganisms for the production of fuels and chemical materials

The possibility of cultivating microorganisms on extremely large scales for the production of single cell protein led to the realization that microorganisms can compete on equal terms with cheap plant products provided that the scale of operation is sufficient. Therefore, there seems little reason why a process for the production of microbial oils and fats should not compete with conventional sources in an attempt to produce a better more cost effective commodity [10].

Historically, the concept of microbial lipids being used as supplementary sources of oils and fats has attracted the maximum attention during the first World Wars due to the disruption of trade and supplies. None of the oil produced, however, was used for edible or even technological purposes.

Its worthed to note that microorganisms display a whole panoply of lipids. The lipid within a cell may vary not only in amount but in type according to how the microorganism is grown, or what stage during growth the microorganism is taken for analysis. Nevertheless, sometimes, they are thought to be more limited in the range and type of lipid which they possess in comparison with animal or plant cells; this is totally incorrect. For example, although microorganisms do not contain prostaglandins or the related leukotrienes and thromboxanes, certain ones do contain the precursors of these molecules - such as PUFAS. However, several researchers have reported the evidence of prostaglandins in yeasts.

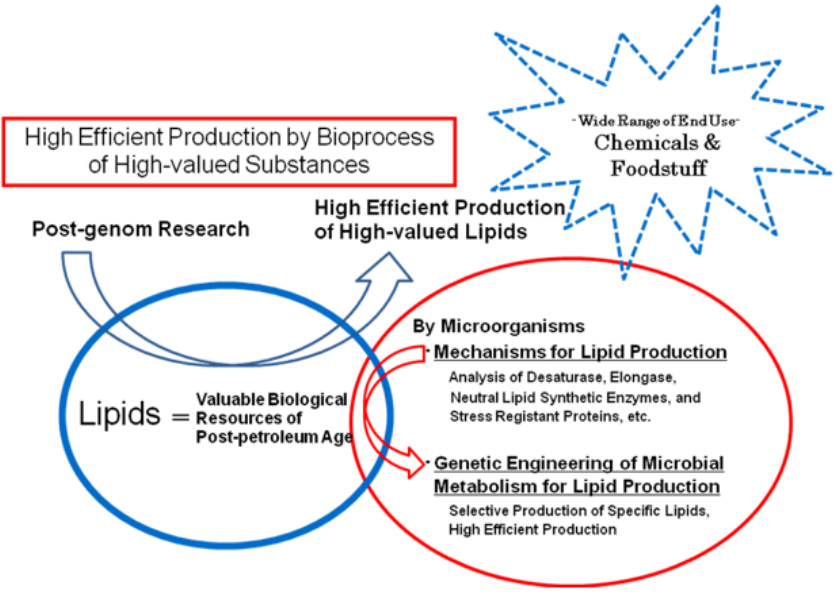

Fig. 1. Analysis of microbial lipid production mechanism and its utilization for high-valued lipid production.

Microbial oils have limited potential as substitutes for plant 
and animal edible oils and fats because they are generally more expensive than agricultural products. Thus, recently, the focus changed from competing with conventional lipid sources to more expensive lipid products with medical applications such as certain polyunsaturated long-chain fatty acids as well as hydroxy polyunsaturated fatty acids, i.e. prostaglandins, leukotrienes and thromboxanes (Fig. 1)

This review aims to examination of oleagenicity and lipid accumulation in filamentous fungi, which are known to possess both $(\omega) 3$ and $(\omega) 6$ series of fatty acids.

\section{DEFINITION AND CLASSIFICATION OF LIPIDS}

Biological materials have been classified as lipids, proteins, carbohydrates and minerals. One of the main characteristics that distinguishes lipids from other natural products is their solubility in organic solvents such as ether, chloroform, benzene, etc. and their insolubility in water. Lipids are hydrophobic or amphipathic small molecules that may originate entirely or in part by carbanion-based condensations of thioesters (fatty acids, polyketides, etc.) and/or by carbocation-based condensations of isoprene units (prenols, sterols, etc.). Lipid classes are; fatty acids, hydrocarbons, acylglycerols, sterols and sterol esters, waxes, phospholipids, glycerophosphingolipids, etherglycerides, sphingolipids and fat-soluble vitamins (A, D, E, K) [11]-[13]. A subdivision into two broad classes, as simple and complex lipids, is convenient for chromatography purposes. Simple lipids are defined as those that on hydrolysis yield at most two types of primary product per mole; complex lipids yield three or more primary hydrolysis products per mole. The complex lipids are best considered in terms of either the glycerophospholipids (or simply if less accurately as phospholipids), which contain a polar phosphorus moiety and a glycerol backbone, or the glycolipids (both glycoglycerolipids and glycosphingolipids), which contain a polar carbohydrate moiety, as these are more easily analyzed separately.

Alternatively, the terms "neutral" and "polar" lipids are used to define these groups, but are less exact [6]. The most common lipid classes in nature consist of fatty acids linked by an ester bond to the trihydric alcohol - glycerol, or to other alcohols such as cholesterol, or by amide bonds to sphingoid bases, or on occasion to other amines. It is the structure of the fatty acid molecules (more correctly fatty acyl chains) attached to the glycerol backbone that accounts for the properties of lipids. The fatty acids are carboxylic acids with an aliphatic tail (chain) synthesized in nature via condensation of malonyl-coenzyme A units by a fatty acid synthetase complex. Fatty acids are important sources of energy because, when metabolized, they yield large quantities of ATP. Most naturally occurring fatty acids have a chain of an even number of carbon atoms from 4 to 28 . The length of the aliphatic chain varies from 2 carbons to 26 carbons; can be short as butyric acid(glycerol) backbone. Lipids, mainly triacylglycerols, are composed of three fatty acids attached to a three carbon properties vary enormously, from hard waxy solids at room temperature (fats) to translucent liquids (oils) [6].

Fatty acids without double bonds are known as "saturated".
Fatty acids that have double bonds are known as "unsaturated", and can have one or more double bonds between carbon atoms. PUFAs contain two or more double bonds. In vivo they exist as fatty acyl constituents of cellular lipids such as triacyiglycerols or phospholipids. Commercial sources of PUFAs are plant/seed oils or fish oils, however, they usually have relatively low PUFA contents. For example, the most successful plant seed oil, Evening Primrose Oil, contains only 9\% w/w $\gamma$-linolenic acid (GLNA), with other sources being black currant oil $(15 \% \mathrm{w} / \mathrm{w}$ GLNA and $18 \%$ w/w $\alpha-18: 3$ (ALNA)) and borage oil (25\% w/w GLNA) [3].

PUFAs are classified into four groups depending on the location of terminal double bond: $(\omega) 3,(\omega) 6,(\omega) 7$ and $(\omega) 9$. The $(\omega) 3$ fatty acids include alpha-linolenic acid which in humans is transformed via eicosapentaenoic acid (EPA) into the 3 -series eicosanoids, i.e. prostaglandins. In contrast, the $(\omega) 6$ series of fatty acids include GLNA, dihomo-y-linolenic acid and arachidonic acid and are eventually transformed to the 1-, 2- and 3-series of eicosanoids in man [14], [15].

It is necessary for either the PUFAs or their precursors to be present in the diet as mammals cannot synthesise all polyunsaturated fatty acids necessary for physiological activities. Mammals can also not desaturate oleic acid to linoleic acid though this is readily carried out by plants, algae and microorganisms. Instead oleic acid is converted to the isomer of linoleic acid which is not found in large amounts in any tissue. Linoleic acid is termed as an essential fatty acid. Liver cells can though desaturate linoleic acid to GLNA acid but not to ALNA acid. Hence, ALNA acid is also an essential fatty acid as it is used for the synthesis of some prostaglandins, leukotrienes and thromboxanes. The biosynthesis of PUFAs in different organisms can involve a variety of pathways, catalyzed by a complex series of desaturation and elongation steps (Fig. 2) [16]-[21]. PUFAs play critical roles in multiple aspects of membrane physiology (i.e. permeability, enzymatic activity) and signaling mechanisms which impact human health and development as well as provide precursors for the biosynthesis of prostaglandins, whose role include mediation of platelet aggregation, influencing blood pressure and stimulation of the immune system.

\section{TRADitional AND Microbial SOURCES OF PUFAS}

Plant seed oils are frequently rich in PUFAs. For example, corn, sunflower, safflower and soya-bean oils are rich in linoleic acid, which may comprise as much as $75 \%$ of the fatty acids present. Fruit/seed oils and foods made from them (i.e. margarines) are important dietary sources of linoleic acid. Some seed oils (i.e. soya-bean oil) also contain ALNA in smaller amounts; green plant tissues are also a source of this fatty acid. The main PUFA in the Western diet is usually linoleic acid followed by $\alpha$-linolenic acid. Fish, especially oily fish (salmon, herring, tuna and mackerel) and fish oils are a rich source of EPA and DHA. In the absence of oily fish or fish oil consumption, ALNA is by far the principal dietary $(\omega) 3$ PUFA [22]-[24]. Microorganisms are being increasingly considered as potential sources of polyunsaturated fatty acids. Early reviews drew the general 
conclusions that ciliate and flagellate protozoa produced no 20 carbon polyunsaturated fatty acids, amoebae lacked C18 polyunsaturated fatty acids, but algae produced the widest range of polyunsaturated fatty acids with fungi usually containing linoleic acid. More recently, attention has focused on certain phylogenetically less-advanced fungi (i.e. Mucor, Phytium and Mortierella spp.) as possible sources of oil because of presence of specific long chain polyunsaturated fatty acids in the oil [25].

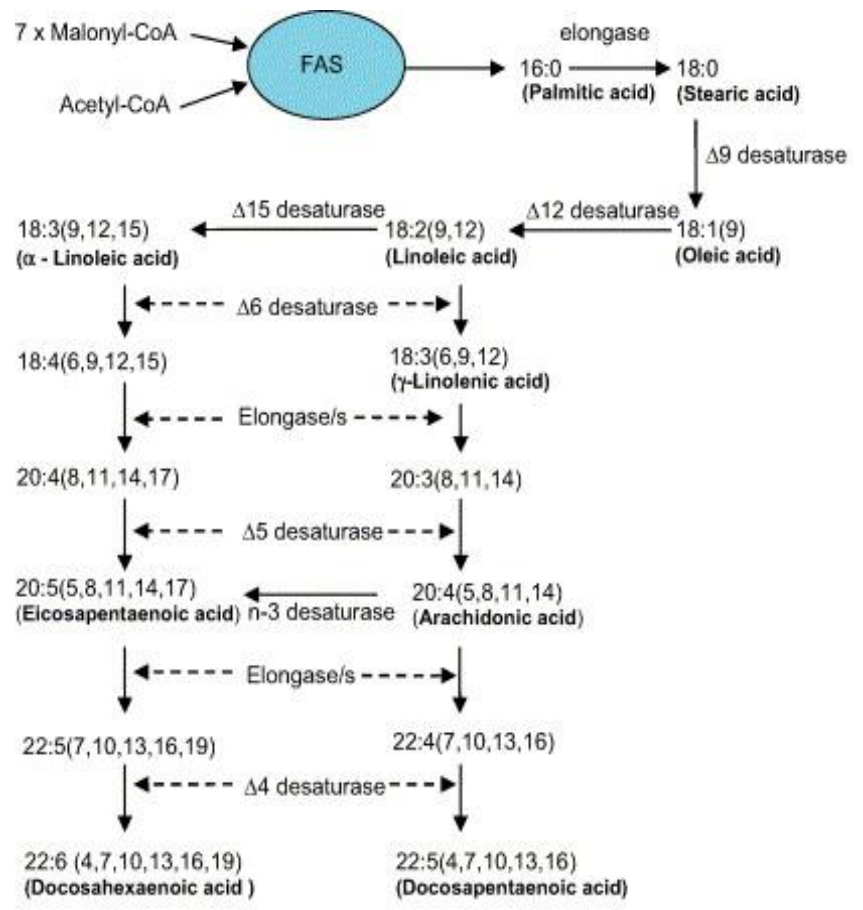

\section{$\mathrm{n}-3$ series $\mathrm{n}-6$ series}

Fig. 2. The biosynthetic routes to polyunsaturated fatty acids and their derivatives.

\section{OLEAGINICITY AND BIOCHEMISTRY OF LIPID ACCUMULATION IN MICROORGANISMS}

Not all microorganisms can be considered as abundant sources of oils and fats, though, like all living cells, microorganisms always contain lipids for the essential functioning of membranes and membranous structures. Organisms, principally eukaryotes, which can accumulate $20 \%$ or more of their biomass as lipid have been termed as "oleaginous" in keeping with oil-bearing plants that are similarly named. Of the some 600 different yeast species, only 25 or so are able to accumulate more than $20 \%$ lipid; of the 60,000 fungal species fewer than 50 accumulate more than $25 \%$ lipid [26], [27].

Biochemical studies suggested a definition for oleaginicity based on the possession of one of the key enzymes for lipogenesis, namely ATP:citrate lyase (ACL). Mother key enzyme involved in lipid accumulation is malic enzyme. ATP:citrate lyase provides the acetyl building units for fatty acid biosynthesis, malic enzyme generates the NADPH by which the acetyl units can be reduced and used as the backbone of the fatty acids. Eukaryotic organisms without ATP:citrate lyase appear unable to achieve the same degree of lipid accumulation as those which possess it. The amount of lipid which an oleaginous microorganism can accumulate is determined by the culture conditions [28].

Lipid accumulates in oleaginous microorganisms when there is an excess of carbon available to the cells during a period when another nutrient which is required for cell proliferation is exhausted from the medium (Fig. 3) [27].

The immediate metabolic consequence of the drop in AMP concentration is a decrease in $\mathrm{NAD}^{+}$isocitrate dehydrogenase activity. The activity of this enzyme in oleaginous cells, unlike non-oleaginous organisms, is dependent completely upon the presence of AMP. As a consequence, isocitrate can no longer be effectively metabolised through the citric acid cycle, causing both isocitrate and citric acid to accumulate. The equilibrium of isocitrate and citrate via acotinase favours citrate. Thus, it is citrate which accumulates in the early period following the onset of nitrogen exhaustion from the medium. As citric acid accumulates, it is transported across the mitochondrial membrane in exchange for malate .Malate serves both as the counter-ion to pyruvate uptake into the mitochondrion and citrate transport out of the mitochondrion. It is also converted back into pyruvate via malic enzyme, an enzyme which produces NADPH to be used subsequently in the biosynthesis of fatty acids. The key to oleaginicity resides not only in the ability of oleaginous organism to accumulate citrate but also subsequently to deal with citrate. Therefore, the principal key to oleaginicity is the possession of ATP:citrate lyase [1].

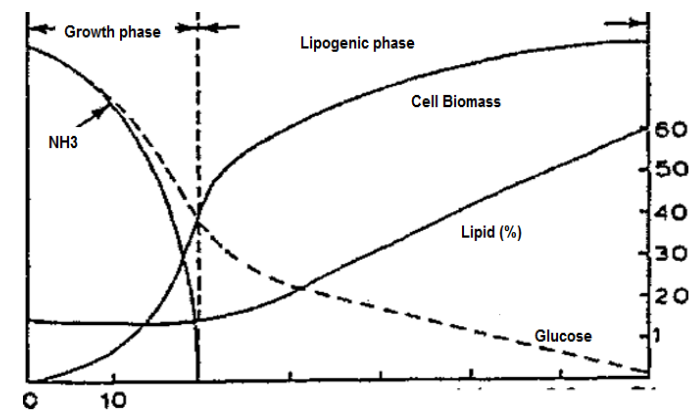

Fig. 3. Typical Lipid accumulation and biomass production pattern in oleaginous microorganisms.

\section{CARbON SOURCES FOR MicROBIAL LIPIDS}

Microorganisms can grow and accumulate lipids on a wide range of carbon sources. The interest is because the composition of accumulated lipid has been stated to reflect the composition of the carbon source. Any carbon source should readily be used by microorganisms. The potential of an organism as an economic producer of lipid is related to the efficiency of substrate utilization. In this connection, many fungi have been screened, particularly for utilization of inexpensive industrial waste products like those from the petroleum industry products, i.e. hydrocarbons. The types of simple hydrocarbons capable of supporting microbial growth as sole sources of carbon and energy are numerous. The most readily assimilated hydrocarbons are straight chain alkalies, such as C10 to C20 n-alkanes. Short-chain (C2 to C8) and long-chain alkanes (C25 to C50) appear to be poorly utilized, even though there are exceptions. It should be noted that there 
is no single organism capable of utilizing all of these carbon sources as growth substrates. In general, any particular strain may utilize a spectrum of hydrocarbons as sole sources of carbon and energy [1].

A large number of researchers have observed that the lipid content of an organism, be it bacteria, yeast or mould, is higher when grown on $n$-alkanes than on non-hydrocarbons. The very growth of a microorganism on a hydrocarbon often leads to an increase in the lipid content of the cell. The reason for increase in lipid content is the slower growth rate of the microorganism on hydrocarbon than on glucose, as the lipid content of an organism often increases at slow growth rates [5].

There is a disadvantage to use them for the production of specific lipid products as one component of the lipid being produced will be an unchanged hydrocarbon substrate. The use of this microbial oil as human or even animal foodstuff would then be difficult. However, there is nothing, to prevent the lipid from being used for technical purposes, such as soaps, detergents, inks, lubricating oils and fats, etc.

Hydrocarbons appear to be natural substrates on which to base a microbial process because they promote lipid synthesis, and even direct the fatty acid composition of the lipid. The realization that many microorganisms could grow as well on alkanes as on glucose suggested microorganisms should also be able to grow on fatty acids and fats, particularly as fatty acids are intermediates in the oxidation of alkanes. The first report of microorganisms cultivated on oils and fats were those in which yeasts were grown on whole fatty fish or fish processing wastes, and subsequently on isolated fish oils [5].

The objectives of the work on microbial utilization of oils and fats have been the production of cheap animal feedstuff and to develop methods for treating and dealing with lipid waste materials from oil- or fat-producing factories. Besides these widely used substrates ubiquitous pentose and hexose sugars are cheap substrates and fungi can grow promptly on these carbon sources. Whey, olive mill wastes, molasses, wheat/rice extracts, orange peel are also other utilized carbon sources.

\section{CUltivation OF FUnGI FOR LIPID PRODUCTION}

For microbial lipid production two fermentation methods are used: 1) Solid-state fermentation and, (the biomolecules are mostly metabolites generated by microorganisms grown on a solid support selected for this purpose) and 2) Submerged fermentation (the sterile medium and mould are added to a large tank where it is constantly mixed and a supply of sterile air is bubbled through (since the fermentation process is aerobic).

Early studies of lipid accumulation were carried out either in batch culture or in two-stage continuous culture. It was generally believed that lipid concentrations equal to those atained in batch culture could not occur in a single stage chemostat. Although the advantages of continuous culture techniques for studying lipid accumulation have ben described, the commercial use of continuous culture is much less evident due to the high capital costs. Therefore, most lipid accumulation studies are conducted with batch fermentations. The usual course of lipid accumulation during batch fermentations can be thought of as a two-stage process: 1) cell proliferation and balanced growth culminating with exhaustion of another nutrient from the medium, and 2) a lipid accumulation phase [29]. The course of lipid accumulation in moulds may be similar to that described for yeasts. Lipid accumulation is a growth-linked process. If the growth rate were to be increased then the rate of lipid accumulation would also increase. The studies on microbial lipid production have shown that the optimum growth conditions are not necessarily best for maximum lipid production. The rate of lipid synthesis relative to the rate of synthesis of other cellular products determines whether lipid accumulates.

\section{FUNGAL LIPIDS}

A fungus is an eukaryote that digests food externally and absorbs nutrients directly through its cell walls. Most fungi reproduce by spores and have a body (thallus) composed of microscopic tubular cells called hyphae. Fungi are heterotrophs and, like animals, obtain their carbon and energy from other organisms. Some fungi obtain their nutrients from a living host (plant or animal) and are called biotrophs; others obtain their nutrients from dead plants or animals and are called saprotrophs (saprophytes, saprobes). Some fungi infect a living host, but kill host cells in order to obtain their nutrients; these are called necrotrophs. Fungi were used to be considered as a member of the plant kingdom, however, its in now classified under the kingdoms of life as eucaryotes [30].

In fungi, lipids occur not only as major constituents of membrane systems, but also as cell wall components, as storage material in abundant and readily observed lipid bodies and, in some cases, as extracellular products. The greater cell-size and complexity of fungi is accompanied by a corresponding diversity of lipid components. The amounts and types of lipid at individual fungal sites vary not only from one organism to another but also with age, stages of development, nutrition and environmental conditions [31]. The lipid content of fungal species can be manipulated by varying culture conditions, therefore, the records of total lipid content are of limited value unless the parameters of growth are specified. More information is available on lipid composition than on any other aspect of fungal lipids, largely because of the ease of preparation and analysis of total lipid samples. The lipid fractions from a variety of moulds, showed wide range of values for the contents of both polar and neutral lipids. Triacylglycerols represent the major lipid component; which are generally considered as storage lipids that may be used for energy and carbon skeletons during growth and development. The other major lipid components of oleaginous moulds are numerous sterols, squalene and other hydrocarbons. There is evidence that sterols exhibit a condensing or liquefying effect on acyl lipids depending on the physical state of the lipid. They may regulate permeability by affecting internal viscosity and molecular motion of lipids in the membrane. They may also serve as precursors of steroid hormones involved in the sexual reproduction of some fungi. Polar lipids of moulds are mainly the phospholipids, but also include glycolipids. Phospholipids are important structural 
components of biological membranes. They have been implicated in the active transport of ions across membranes and are also essential for the activity of some membrane-bound enzymes [32], [33]. The relative proportions of the all the lipid components may vary according to the stage of fungal development, age and conditions under which fungus is cultured. The advantages of using moulds rather than yeasts to accumulate lipid must be seen either in the ability of moulds to handle and upgrade a greater range of waste materials than yeasts or in their ability to produce a wider diversity of fatty acids. The majority of fungal species contain, in order of abundance, oleic acid (C18:1), palmitic acid (C16:0) and linoleic acid (C18:2) as the major acids, with stearic acid (C18:0), linolenic acid (C18:3) and palmitoleic acid (C16:1) as the minor ones. Besides the occurrence of high amounts of PUFAs, C18:2 and C18:3, Mucorales uniquely contain $\gamma$-linolenic acid (C18:3, $\mathrm{n}-6)$ rather than $\alpha$-linolenic acid. In addition arachidonic acid (C20:4), eicosapentaenoic acid (C20:5) and docosahexanoic acid (C22: 5) occur in the same species [27], [34]-[36].

\section{CURRENT APPLICATIONS FOR FUNGAL LIPIDS}

In recent years, interest in microbial lipids has been renewed because of an urgent need for prudent utilization of alternative renewable resources as carbon sources for production of lipids and because of medical and nutritional research has revealed potential uses of PUFAs for practical applications. Polyunsaturated fatty acids are known to have important roles when present in the diet rather than administered as pseudo-drugs. The low incidence of heart disease in Iceland, Eskimo and some Japan population has been attributed to the high proportions of marine fish in their diet, which has various polyunsaturated fatty acids [32]. To date only three PUFAs have been produced commercially using microorganisms. The first was SCO rich in GLNA produced using Mucor circinelloides, and the others were SCO rich in ARA and DHA produced using Mortierella spp. GLNA has long been regarded as a "cure-all", which is best found in evening primrose seed oil used for the treatment of eczema. The oil has also been shown to aid sufferers of chronic inflammatory arthritis. GLNA is effective in diseases such as Alzheimer, cancer, depression, peroxisomal disorders, hyperactivity. The $(\omega) 6$ polyunsaturated fatty acids, dihomo- $\gamma$-linolenic) and arachidonic acid have very important roles in mammalian biochemistry as precursors of prostaglandins. The $(\omega) 3$ polyunsaturated fatty acids, eicosapentaenoic acid and docosahexaenoic acid have been shown to reduce the occurrence of cardiovascular disease and inflammatory disease such as asthma and type I diabetes mellitus [37]-[40]. From biotechnological viewpoint, it is the high content of the polyunsaturated fatty acids that has singled out fungi for recent attention.

\section{REFERENCES}

[1] A. Akpinar, "Transformations of fatty acids in filamentous fungi," Ph.D. dissertation, Dept. Biolog. Sci., University of Hull, Hull, UK, 1997.

[2] F. D. Gunstone, J. L. Harwood, and F. B. Padley, The Lipid Handbook, 2nd ed. Chapman and Hall, NY, 1994, pp. 202-225.
[3] M. I. Gurr, J. L. Harwood, and K. N. Frayn, Lipid Biochemistry: An Inroduction, 5th ed. John Wiley\&Sons, 2008, pp. 13-92.

[4] D. Voet and J. G. Voet, Biochemistry, 5th Ed. John Wiley\&Sons, 2011.

[5] C. Ratledge and S. G. Wilkinson, "An overview of microbial lipids," Microbial Lipids, Kluwer Academic Publishers, London, 1988, vol. 1, pp. 89-141.

[6] W. W. Christie, Lipid Analysis, 3rd ed. The Oily Press, Ayr, Scotland, 2003.

[7] H. Sprecher, "Metabolism of highly unsaturated n-3 and n-6 fatty acids," Biochimica Biophysica Acta, vol. 1486, pp. 219-231, 2000.

[8] A. Akpinar-Bayizit and T. Ozcan-Yilsay, "Peyniraltı suyundan mikrobiyel yağ üretimi (in Turkish)," Akademik Glda, vol. 2, pp. 15-17, 2004.

[9] D. Suddaby, Essential Fatty Acids: a review of their biochemistry, function, interaction and clinical applications, Croda Universal Ltd., Hull, 1992.

[10] S. Papanikolaou, M. Galiotou-Panayotou, S. Fakas, M. Komaitis, and G. Aggelis, "Lipid production by oleaginous Mucorales cultivated on renewable carbon sources," Eur. J. Lipid Sci. Technol., vol. 109, pp. 1060-1070, 2007.

[11] K. Larsson, P. Quinn, K. Sato, and F. Tiberg, Lipids: Structure, Physical Properties and Functionality, vol. 19, The Oily Press, PJ Barnes and Associates, Bridgwater, UK, 2006, pp. 1-5.

[12] G. Di Paolo and M. R. Wenk, Lipids, Academic Press, 2012.

[13] R. A. Harvey and D. R. Ferrier, Biochemistry, Lippincott Williams \& Wilkins, 2011

[14] M. Castillo, F. Amalik, A. Linears, and E, Garcia-Perogrin, "Fish oil reduces cholesterol and arachidonic acid levels in plasma and lipoproteins from hypercholesterolemic chicks," Molecular and Cellular Biochemistry, vol. 210, pp. 121-130, 2000.

[15] D. A. Hughes and A. C. Pinder, "N-3 Polyunsaturated fatty acids inhibit the antigen-presenting function of human monocytes," American Journal of Clinical Nutrition, vol. 71, pp. 357-360, 2000.

[16] M. J. James, R. A. Gibson, and L. G. Cleland, "Dietary polysaturated fatty acids and inflammatory mediator production," American Journal of Clinical Nutrition, vol. 71, pp. 3438-3488, 2000.

[17] C. Von Schacky, "n-3 fatty acids and the prevention of coronary atherosclerosis," American Journal of Clinical Nutrition, vol. 71, pp 224-227, 2000.

[18] T. Yamada, J. P. Strong, T. Ishii, T. Ueno, M. Koyama, H. Wagayama, A. Shimizu, T. Sakai, G. T. Malcom, and M. A. Guzman, "Atherosclerosis and $\omega 3$ fatty acids in the populations of a fishing village and a farming village in Japan," Atherosclerosis, vol. 53, pp. 469-481, 2000.

[19] A. Leaf, Y. F. Xiao, J. X. Kang, and G. E. Billman, "Prevention of sudden cardiac death by $n-3$ polyunsaturated fatty acids," Pharmacology and Therapeutics, vol. 98, pp. 355-377, 2003.

[20] J. A. Nettleton and R. Katz, "n-3 long-chain polyunsaturated fatty acids in type II diabetes: A review," Journal of the American Dietetic Association, vol. 105, pp. 428-440, 2005.

[21] E. B. Schmidt, L. H. Rasmussen, J. G. Rasmussen, A. M. Joensen, M B. Madsen, and J. H. Christensen, "Fish, Marine n-3 polyunsaturated fatty acids and coronary heart disease:A minireview with focus on clinical trial data," Prostaglandins, Leukotrienes and Essential Fatty Acids, vol. 74, pp. 17-21, 2006.

[22] A. Ascherio, E. B. Rimm, M. J. Stampfer, E. L. Giovannucci, and W. C. Willett, "Dietary intake of marine n-3 fatty acids, fish intake, and the risk of coronary disease among men," New England Journal of Medicine, vol. 332, pp. 977-983, 1995.

[23] E. Mantzioris, L. G. Cleland, R. A. Gibson, M. A. Neumann, M. Demais, and M. J. James, "Biochemical effects of a diet containing foods enriched with n-3 fatty acids," American Journal of Clinical Nutrition, vol. 72, 42-48, 2000.

[24] J. M. Kremer, "n-3 fatty acid supplements in rheumatoid arthritis," American Journal of Clinical Nutrition, vol. 71, pp. 349-351, 2000.

[25] M. Certik, and S. Shimizu, "Biosynthesis and regulation of microbial polyunsaturated fatty acid production," Journal of Bioscience and Bioengineering, vol. 87, pp. 1-14, 1999.

[26] C. Ratledge and S. G. Wilkinson, Microbial Lipids, vol. 2, Academic Press, London, 1989.

[27] C. Ratledge, "Microorganisms as sources of polyunsaturated fatty acids," in Structured and Modified Lipids, F. D. Gunstone, Ed. Marcel Dekker, NY, 2001, pp 351-399.

[28] J. P. Wynn, A. A. Hamid, and C. Ratledge, "Biochemical events leading to the diversion of carbon into storage lipids in the oleaginous fungi Mucor circinelloides and Mortierella alpine," Microbiology, vol. 147, pp. 2857-2864, 2001. 
[29] A. Anderson, and J. P. Wynn, "Microbial Polyhydroxyalkanoates, Poysaccaharides and Lipids," in Basic Biotechnology, C. Ratledge, B. Kristiansen, Eds. 2nd edition, Cambridge University Press, Cambridge, 2001, pp 325-348.

[30] J. D. Weete, Lipid biochemistry of fungi and other organisms, Plenum press, London, 1980.

[31] J. D. Weete and D. J. Weber, Lipid Biochemistry of Fungi and Other Organisms, Plenum Press, 1980.

[32] M. Sancholle, F. Laurelle, D. M. Lösel, and J. Muchembled, "Biotechnological potential of fungal lipids," in Handbbok of Fungal Biotechnology, D. K. Arora, Ed. CRC Press, 2003, pp. 586-606.

[33] G. N. Cohen, Microbila Biochemistry, Springer, 2011.

[34] T. Nakahara, T. Yokochi, Y. Kamisaka, and O. Suzuki, " $\gamma$-Linolenic Acid from the Fungus Mortierella," in Industrial Appilications of Single-cell Oils, D. J. Kyle, C. Ratledge, Eds. American Oil Chemists' Society, Champaign, Illinois, 1992, pp. 61-97.

[35] M. Pillai, A. Ahmad, T. Yokochi, T. Nakahara, and Y. Kamisaka, "Biosynthesis of triacylglycerol molecular species in an oleaginous fungus Mortieralla ramanniana var. angulispora," Journal of Biochemistry, vol. 128, pp. 121-126, 2002.

[36] O. P. Ward and A. Singh, "Omega-3/6 fatty acids: Alternative sources of production," Process Biochemistry, vol. 40, pp. 3627-3652, 2005.

[37] J. P. Carter, "Gamma-Linolenic acid as a nutrient," Food Technology, vol. 42, pp. 72-82, 1988.
[38] L. Oxlade, "Kings cure-all makes a comeback," Chemistry in Britain, vol. 26, 1990.

[39] I. S. Newton, "Polyunsaturated fatty acids in diet and health," Chemistry and Industry, vol. 8, pp. 302-305, 1997.

[40] M. Dong and T. H. Walker, "Addition of polyunsaturated fatty acids to canola oil by fungal conversion," Enzyme and Microbial Technology, vol. 42, pp. 514-520, 2000.

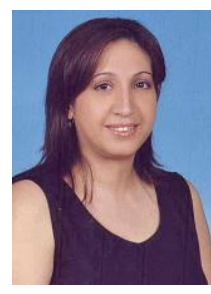

Arzu Akpinar-Bayizit is an assistant professor at the Department of Food Engineering, Uludag University, Bursa/Turkey. After having M.Sc. degree at Uludag University on 1994, she had her Ph.D. degree at the Department of Biological Sciences of the University of Hull, United Kingdom, on 1997. The topic of her Ph.D. project funded by Higher Education Council of Turkey was on fungal lipid metabolism and a novel fatty acid was identified from a sewage fungus.

The main lectures given by Mrs. Akpinar-Bayizit are instrumental analysis, microbial process technology, food fermentations, functional foods and plant hygiene and sanitation. Her areas of her research interest include fermentation technology, particularly microbial fermentations, and lipid technology. Up till today she has supervised 5 M.Sc. studies, and supervising ongoing 4 M.Sc. and 2 Ph.D. projects. She has published several research and review articles in international journals and has two book chapters. 